ORIGINAL RESEARCH

\title{
Mt Everest Base Camp Medical Clinic "Everest ER": Epidemiology of Medical Events During the First 10 Years of Operation
}

\author{
Mária Némethy, MD; Andrew B. Pressman, MD; Luanne Freer, MD; Scott E. McIntosh, MD, MPH \\ From the Division of Emergency Medicine, University of Utah, Salt Lake City, UT (Drs Némethy and McIntosh); the Department of Emergency \\ Medicine, William Beaumont Hospital, Royal Oak, MI (Dr Pressman); and the Everest ER, Nepal, and Yellowstone National Park, WY (Dr Freer).
}

Objectives. - As the highest peak on the planet, Mt Everest provides a truly austere environment in which to practice medicine. We examined records of all visits to the Everest Base Camp Medical Clinic (Everest ER) to characterize the medical problems that occur in these patients.

Methods.-A retrospective analysis of medical records from the first 10 years of operation (2003 to 2012) was performed.

Results.-Medical reasons accounted for $85.3 \%$ (3045) of diagnoses, whereas $14.0 \%$ (500) were for trauma. The most common medical diagnoses were pulmonary causes such as high altitude cough and upper respiratory infection, comprising more than $38 \%$ of medical diagnoses. For traumatic diagnoses, $56 \%$ were for dermatologic causes, most commonly for frostbite and lacerations. Pulmonary and dermatologic diagnoses were also the most frequent causes for evacuation from Everest Base Camp, most commonly for high altitude pulmonary edema and frostbite, respectively.

Conclusions.-Medical professionals treating patients at extreme altitude should have a broad scope of practice and be well prepared to deal with serious trauma from falls, cold exposure injuries, and altitude illness.

Key words: Everest, injury, illness, trauma, clinic, mountaineering

\section{Introduction}

More than 800 climbers or hired climbing staff attempt to summit Mt Everest every year. With an elevation of 8848 m (29,029 feet), Everest's peak has a barometric pressure of $253 \mathrm{~mm} \mathrm{Hg}$ and an ambient $\mathrm{PO}_{2}$ of $53 \mathrm{~mm}$ $\mathrm{Hg}$. These values are roughly one-third those at sea level, where barometric pressure is $760 \mathrm{~mm} \mathrm{Hg}$ and $\mathrm{Po}_{2}$ is 159 $\mathrm{mm} \mathrm{Hg}$. ${ }^{1}$ Compared with the 13 other $8000-\mathrm{m}$ peaks, $\mathrm{Mt}$ Everest's summit is attempted more often and has more total ascents. By the end of the 2012 spring climbing season, 5208 total ascents of Mt Everest had been made. Comparatively, the next most popular 8000-m peak, Cho Oyu, had 3171 ascents during the same time period. ${ }^{2}$

The Everest Base Camp (EBC) Medical Clinic (Everest ER) was established in 2003 in cooperation with the

Disclosure: The authors have no conflicts of interest to disclose.

Corresponding author: Andrew B. Pressman, MD, Department of Emergency Medicine, William Beaumont Hospital, 3601 West 13 Mile Road, Royal Oak, MI 48073 (e-mail: andrew.pressman@beaumont. edu).
Himalayan Rescue Association (HRA) to provide medical care to the climbers, support staff, and visitors to this uniquely austere environment. Since 2003, the Everest ER has been staffed yearly during the busiest climbing months of April and May. Located at the base camp within Nepal, the Everest ER is located at an elevation of $5364 \mathrm{~m}(17,598$ feet $)$ and has an ambient barometric pressure of $400 \mathrm{~mm} \mathrm{Hg}$ and $\mathrm{Po}_{2}$ of $84 \mathrm{~mm} \mathrm{Hg} .{ }^{3}$ The clinic's mission is to treat climbers attempting the summit and foreign trekkers to EBC, using proceeds from this care to subsidize free or low-cost health care to local Nepalese.

The Everest ER is staffed by a minimum of 2 physicians who have worked at least 1 prior season in one of the other HRA clinics, located in Pheriche and Manang. In 2009, a Nepali physician position was added to the staff. In addition, physicians participate in rescue and medical radio calls to assist climbers at higher elevations on the mountain. However, with advances in helicopter technology, direct evacuations of more acute patients from higher elevations have recently been 
accomplished, in some cases without the patient ever being treated at the Everest ER.

The Everest ER consists of a 3.7-m by 6.1-m doublewalled tent erected every April and removed at the end of the spring climbing season in May. The clinic uses a separate 8-person dome tent as a waiting room or treatment area for minor complaints. The Everest ER can run entirely on solar energy, and relies on a backup generator as needed. In addition to a robust medication formulary that includes resuscitation medications, the Everest ER offers oxygen cylinders, an oxygen concentrator, 12-lead electrocardiograph, transport monitors, basic suturing equipment, portable hyperbaric chambers, and needle thoracostomy equipment. Figure 1 shows the exterior and interior of the main tent.

Currently, the only available information on patterns of disease from Mt Everest is from data released after the first season of the Everest $\mathrm{ER}^{4}$; no longitudinal data have been previously reported. We examined records of visits to the Everest ER to characterize the medical problems that occur in these patients to prepare future medical professionals who care for patients in this type of environment.

\section{Methods}

Clinic documentation was reviewed for patients seen at the Everest ER during its first 10 consecutive seasons (2003 to 2012). Recorded information included patient demographics, diagnoses, treatments, prescriptions, medications dispensed, and evacuation type, if any. From 2003 to 2008, all original documentation was handwritten and then transferred to a computerized spreadsheet at the end of the climbing season. From 2009 to
2011, the transfer from the handwritten documentation to spreadsheet occurred in real time on a computer in the Everest ER. From 2012 onward, documentation has used an iPad application, which is then extracted into the spreadsheet. Radio consults or evacuations at higher elevations were not included in documentation and, therefore, were not included in the analysis.

If the patient had multiple diagnoses, each diagnosis was included individually. If not explicitly recorded, the category of diagnosis (medical vs trauma) was inferred from the diagnosis. Diagnoses were then grouped into the categories listed in Table 1 that are based on the 14 recognized systems by the US Center for Medicaid and Medicare Services. In addition to these 14 systems, we also included dental, toxicology, and a general category for visits that incorporated general advice, checkups, medication refills, and vital signs checks. Records that were incomplete or illegible were included in the analysis as missing data.

Because of the Everest ER's unique environment for altitude- and cold-related complaints, subgroup analysis was done specifically on altitude-associated conditions and cold exposure injury. We defined cold exposure as frostbite, frost nip, and nonfreezing cold injury. We included acute mountain sickness (AMS), high altitude pulmonary edema (HAPE), high altitude cerebral edema (HACE), and periodic breathing as altitude-associated conditions.

As there are no recorded data for the total number of EBC visits annually, all percentages included represent proportions of the total diagnoses and do not represent prevalence or incidence. Descriptive information was analyzed with SPSS (version 20; IBM Inc, Chicago, IL). Ethical approval was obtained from the University of

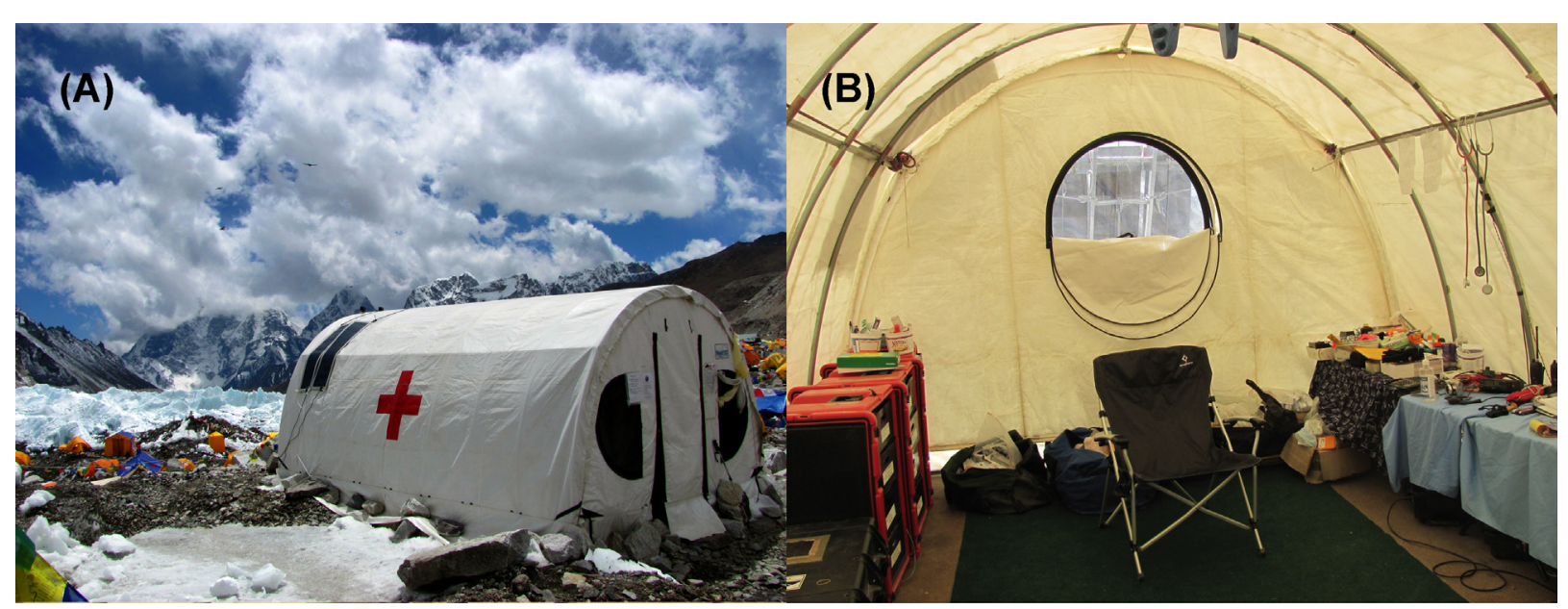

Figure 1. Photographs of the (a) exterior and (b) interior of the Everest ER. Photographs by Magnus Johansson. 
Table 1. Categories of medical diagnoses seen at the Everest ER, 2003-2012

\begin{tabular}{lrc}
\hline Diagnosis category & Number & Percent \\
\hline Pulmonary & 1164 & 38.2 \\
Gastrointestinal & 480 & 15.8 \\
Ear, nose, throat & 439 & 14.4 \\
Neurology & 199 & 6.5 \\
Musculoskeletal & 144 & 4.7 \\
Constitutional & 139 & 4.6 \\
Dermatology & 106 & 3.5 \\
General & 81 & 2.7 \\
Dental & 70 & 2.3 \\
Eye & 51 & 1.7 \\
Cardiovascular & 50 & 1.6 \\
Psychiatric & 42 & 1.4 \\
Genitourinary & 40 & 1.3 \\
Allergic/immunologic & 15 & 0.5 \\
Endocrine & 13 & 0.4 \\
Toxicology & 11 & 0.4 \\
Hematologic/lymphatic & 1 & 0 \\
Total & 3045 & \\
\hline
\end{tabular}

Missing data $=24(0.7 \%)$.

Utah Institutional Review Board and the Nepal Health Research Council.

\section{Results}

During the spring climbing seasons of 2003 through 2012, physicians at the Everest ER treated 2941 patients, with a total of 3569 diagnoses (missing data for 24 patients when diagnosis was not recorded). Of all patients seen, $83.2 \%$ (2447) were male and $8.9 \%$ (262) were female (missing data, 232). The average age for patients seen was 37.2 years old $(\mathrm{SD} \pm 11.3$ years; range, 13 to 87 years). Nearly 53\% (1548) of patients were seen in April, and 40.4\% (1189) were seen in May. The remaining $6.9 \%$ (204) of patients did not have a date recorded. Of all patients seen, 97.1\% (2856) were climbers or climbing-related staff, and only $2.9 \%$ (85) were trekkers. The number of patient visits per climbing season increased each year compared with the total numbers of climbers on Mt Everest for the corresponding years as shown in Figure 2. Of the physicians staffing the Everest ER during this time, 9 were emergency physicians, 7 were general practitioners, and 1 was a general surgeon.

The frequencies of medical diagnosis categories are shown in Table 1, and the frequencies of the most common traumatic diagnoses are listed in Table 2. Medical reasons accounted for $85.3 \%$ (3045) of diagnoses, and $14.0 \%$ (500) were for trauma. The nationality of patients seen at the Everest ER is included in Table 3.

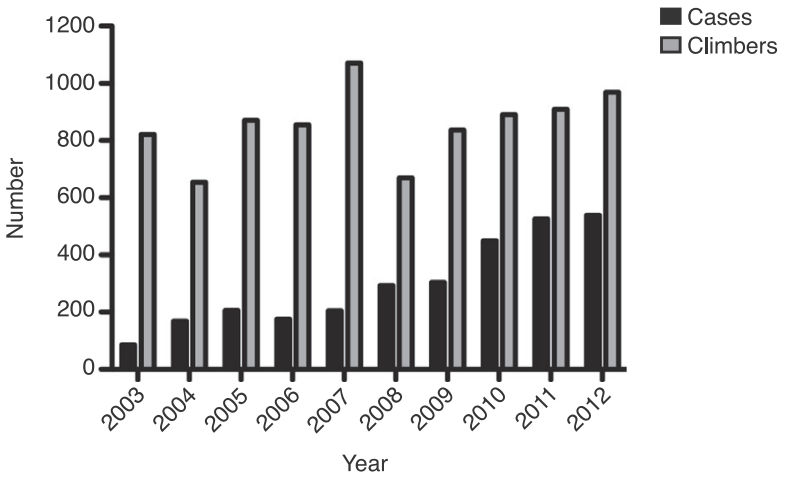

Figure 2. Number of cases treated each climbing season compared with total climbers, 2003-2012. Annual climber data includes both expedition members and climbing support staff above base camp.

The most common medical diagnostic category was pulmonary disease. There were a total of 1165 pulmonary diagnoses with only 1 case (a pneumothorax) caused by trauma. The most common medical diagnosis within the pulmonary category was high altitude cough, accounting for $37.2 \%$ of pulmonary diagnoses. The next most common diagnosis in the pulmonary category was upper respiratory infection, accounting for $15.3 \%$ of pulmonary diagnoses. Bronchitis and cough of uncertain etiology accounted for $12.3 \%$ and $12 \%$ of pulmonary diagnoses, respectively. Uncommon but interesting respiratory diagnoses included 2 presumed diagnoses of pulmonary embolism and 2 possible pulmonary tuberculosis infections.

The second most common medical diagnosis category seen at the Everest ER was gastrointestinal disease. The most common diagnosis within the gastrointestinal category was acid reflux, accounting for $35.8 \%$ of gastrointestinal diagnoses. The next most common gastrointestinal diagnoses were diarrhea and gastroenteritis, accounting for $25.6 \%$ and $13.1 \%$ of all gastrointestinal diagnoses, respectively. Higher-acuity gastrointestinal diagnoses included 3 cases of acute

Table 2. Categories of traumatic diagnoses seen at the Everest ER, 2003-2012

\begin{tabular}{lrc}
\hline Diagnosis category & Number & Percent \\
\hline Dermatology & 280 & 56 \\
Musculoskeletal & 175 & 35 \\
Eye & 22 & 4.4 \\
Dental & 12 & 2.4 \\
Neurology & 7 & 1.4 \\
Ear, nose, throat & 3 & 0.6 \\
Pulmonary & 1 & 0.2 \\
Total & 500 & 100 \\
\hline
\end{tabular}

Missing data $=24(4.8 \%)$. 
Table 3. Nationalities of patients seen at the Everest ER, 20032012

\begin{tabular}{lrc}
\hline Country & Number & Percent \\
\hline Nepal & 1499 & 51 \\
United States & 590 & 20.1 \\
United Kingdom & 113 & 3.8 \\
Canada & 87 & 3 \\
Australia & 80 & 2.7 \\
India & 73 & 2.5 \\
Argentina & 39 & 1.3 \\
Korea & 38 & 1.3 \\
Mexico & 31 & 1 \\
Other & 360 & 12.2 \\
\end{tabular}

Missing data $=31(1 \%)$

abdomen, 3 cases of acute cholecystitis, and 1 case of Crohn's disease exacerbation.

Less common but notable medical diagnoses included 9 cases of acute coronary syndrome with 1 documented case of acute myocardial infarction, 1 case of adrenal crisis, and 1 case of cauda equina syndrome.

When considering traumatic disease, the most common diagnoses were from the dermatologic category. The most common dermatologic trauma was from frostbite, which accounted for $27.5 \%$ of all traumatic dermatologic complaints. Lacerations accounted for $20.7 \%$ and contusions for $12.1 \%$ of traumatic dermatology diagnoses. Medical dermatologic complaints were not nearly as common as traumatic ones, but included 3 cases of herpes zoster as well as numerous cases of cellulitis $(n=21)$ and abscesses $(n=25)$. Abscess locations included 4 scrotal and 4 perirectal abscesses.

The second most common traumatic category was for musculoskeletal diagnoses. Ankle injury accounted for $14.8 \%$ of all musculoskeletal traumas followed by back injury (13.1\%), intercostal injury (8.6\%), and knee injury $(8.0 \%)$. Although fractures were less common than strains, notable fractures included 2 tibia/fibula fractures and 1 pelvis fracture.

In subgroup analysis, altitude-associated conditions accounted for only $6.7 \%$ of all medical diagnoses. Of all altitude illness seen at the Everest ER, $46.8 \%$ were for AMS, $35.6 \%$ were for HAPE, $9.3 \%$ were for HACE, and $8.3 \%$ were for periodic breathing. Cold exposure accounted for $18.4 \%$ of all trauma visits. Cold injuries consisted of $83.7 \%$ for frostbite, $10.9 \%$ for frost nip, and $5.4 \%$ for nonfreezing cold injury.

Of the 2941 patients seen, 180 were advised to evacuate, of whom 2 refused evacuation. Fifty-four patients (30\%) required helicopter evacuation, 56 patients $(31.1 \%)$ were ambulatory for ground evacuation,

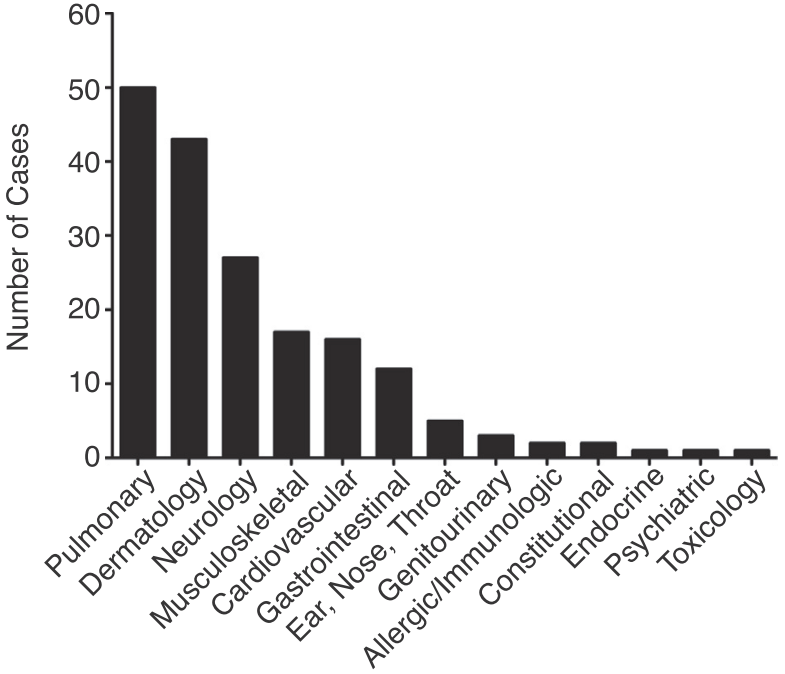

Figure 3. Number of diagnosis categories requiring evacuation from the Everest ER, 2003-2012.

and 1 patient $(0.5 \%)$ was evacuated by horse. Sixtyseven additional patients (37.2\%) also required evacuation, but no mode of transport was documented. The most common diagnosis categories for patients requiring evacuation are included in Figure 3. Specifically, the top 3 diagnoses requiring evacuation were 33 cases of frostbite $(18.3 \%), 33$ cases of HAPE $(18.3 \%)$, and 10 cases of HACE $(5.6 \%)$.

\section{Discussion}

Everest ER sees a wide variety of illnesses and injuries, most frequently pulmonary, ear/nose/throat, and gastrointestinal complaints. Although the vast majority of diagnoses could be treated at EBC, the most common complaints requiring evacuation were caused by either altitude-related illness or cold exposure injury.

As can be seen in Figure 2, the rate of growth in patient volume seen at the Everest ER has outpaced the rate of growth of climbers on Mt Everest in the last decade. Everest ER utilization may be increasing as a result of growing awareness of and perceived physician expertise available at the clinic. As was reported after its first year, the Everest ER has continued to treat more Nepalese patients than foreigners. ${ }^{4}$ The Everest ER and HRA's mission to provide free or subsidized low-cost health care to the Nepali people of the Everest region is clearly met, as the distribution of nationalities treated at the Everest ER affirms.

Our findings are consistent with earlier studies that found a wide variety of complaints seen at high-altitude clinics. ${ }^{5,6}$ Although the most common diagnosis types seen at the Everest ER were pulmonary, ear/nose/throat, and gastrointestinal diagnoses, these same categories 
made up a lower proportion of diagnoses seen at a highaltitude clinic on Denali. ${ }^{7}$ Clinicians at Everest ER may be treating a broader patient population for a longer duration with a wider variety of complaints than on Denali. These findings suggest that it would be important to staff similar clinics in the future with clinicians with a wide scope of practice in addition to expertise in cold exposure and altitude-related diseases. When so far from definitive care, Everest ER physicians must be able to accommodate and treat injuries and illnesses that many medical clinics might be ill prepared to manage, from primary-care issues to trauma. Although the majority of diagnoses seen at the Everest ER in its first decade were of lower acuity, the Everest ER has also diagnosed cauda equina syndrome, acute myocardial infarctions, tachyarrhythmias, sepsis, pulmonary emboli, strokes, head injuries, and pelvis fracture.

The most frequent diagnosis seen from 2003 to 2012 was high altitude cough, a poorly understood and illdefined diagnosis. The cause of high altitude cough remains unknown, and some experts propose the etiology to be bronchial irritation from inhaling cold air with very low humidity. Recently, however, this has been called into question, and high altitude cough may encompass several different processes including bronchoconstriction, respiratory water loss, respiratory tract infections, rhinitis, and postnasal drip. ${ }^{8,9}$ The prevalence of high altitude cough at the Everest ER is dramatically different from what has been described at Denali from 1992 to 2011. High altitude cough was responsible for $11.5 \%$ and undifferentiated cough for $3.7 \%$ of diagnoses seen at the Everest ER. On Denali, on the other hand, there were no diagnoses of high altitude cough, and undifferentiated cough was only $0.5 \%$ of diagnoses reported from Denali. ${ }^{7}$

The difference in diagnosis patterns of high altitude cough between Denali and Mt Everest may have multiple explanations. It could possibly be related to the higher altitude found at Everest, with longer acclimatization requirements and, thus, longer time spent at extreme elevations. Another possible explanation could be increased exposure to viral illness from interaction with trekkers along the trail, where it is common for both of these groups to live in close quarters. Comparatively, nonclimbers rarely mix with climbers in Denali, as it requires flight access. The high altitude cough found in the Everest region may also originate from inhaled particulates encountered before reaching EBC. Emissions from traffic in Kathmandu, as well as dung-burning stoves in the Himalaya, have been linked to poor air quality and negative respiratory effects. ${ }^{10-13}$ Furthermore, as more than $40 \%$ of the diagnosed high altitude cough occurred in Nepalese patients, it is possible that living with this same poor air quality chronically could make these patients more susceptible.

In regards to minor dermatologic traumas, the proportion of diagnoses for lacerations, contusions, and blisters was nearly identical between Denali and Mt Everest. $^{7}$ As would be expected, the high proportion of minor dermatologic and musculoskeletal traumas are also consistent with multiple studies of wilderness traumas at lower elevations. ${ }^{14,15}$ Although these findings are not surprising, they further reinforce efforts to educate climbers going to extreme altitude about the need to be prepared for minor injuries. The ramifications of such minor injuries in an environment as austere as Mt Everest may be more serious than in other venues, and climbers should be adept at minor first aid and carry adequate supplies in personal medical kits. More serious traumatic injuries were usually associated with falls, which previously have been identified as a leading cause of serious mountaineering trauma. ${ }^{16,17}$

Although the majority of diagnoses seen at the Everest ER were for relatively minor complaints, the most common reasons for evacuation were for altituderelated conditions or cold exposure injury. This was expected, as altitude illness is found in substantial numbers even at much lower elevations than those found at EBC and on Mt Everest. ${ }^{18-20}$ Although AMS was the most commonly diagnosed altitude-related condition, HACE and HAPE were far more likely to result in evacuation. This seemingly obvious finding emphasizes the importance in distinguishing AMS from early HACE and in equipping any similar clinics in the future with adequate means to diagnose and evacuate patients with these serious conditions.

Cold exposure injuries were also an important cause for evacuation from EBC. This findings is of little surprise, as it is consistent with findings from other mountaineering regions from around the world., ${ }^{7,21,22}$ Although frostbite accounted for a relatively small proportion of the total diagnoses seen at the Everest ER, it has recently been estimated that the average time it takes facial flesh to freeze on the peak during the spring climbing season is only 5 minutes. ${ }^{23}$ This implies that most climbers were either taking adequate precautions to avoid injury or were not seen at the clinic because of self-treatment, direct evacuation, or underreporting. As such, it is extremely important for future practitioners and other mountaineering clinics to be adequately prepared to treat and evacuate severe cold exposure injuries.

The results of our study indicate that high altitude medical professionals should be competent in treating a very wide array of medical illnesses and traumas. The unique high altitude environment also requires that 
physicians should be able to treat and assist in evacuating patients with altitude illness and cold exposure injuries. Further research could seek to estimate total EBC visits as well as disease treated by expeditions to better estimate prevalence and incidence of disease. Also, it appears that the Everest region of Nepal may be a unique environment to study further the pathophysiology of HACE, as it is found comparatively more frequently in the area.

\section{LIMITATIONS}

The data collected for this study were retrospective and limited to the original documentation. Varied diagnosing among clinicians throughout the years made analysis of specific diagnoses difficult. This resulted in many distinct diagnoses having overlap, such as cough in addition to high altitude cough or gastrointestinal upset in addition to dyspepsia, gastroenteritis, nausea/vomiting, and diarrhea. Individual variations in methods of documentation and year-to-year documentation variability led to inconsistencies in data collection. For example, the sex of patients was not documented for all of 2005, although it was for the other years. In addition, the clinicians were practicing with limited diagnostic tools, leaving definitive diagnosis in question at times. When patients were evacuated to definitive care, diagnosis follow-up was usually obtained. However, for most other patients, diagnosis was not confirmed if further testing or imaging was required to do so. Finally, there is no available record of the total number of visits that the EBC receives annually or diagnoses treated by individual expedition clinicians. Because of these limitations, no accurate estimate of incidence or prevalence of disease at the EBC can be drawn from the current data.

\section{Conclusions}

The Everest ER sees a wide array of patients and diagnoses. Although the majority of complaints are for minor problems such as high altitude cough and dermatologic traumas, numerous cases of life-threatening illnesses do occur. Climbers and medical professionals should be prepared for a wide array of illness, as well as be educated and well equipped to deal with lifethreatening falls, frostbite, and severe altitude-related disease.

\section{Acknowledgments}

The authors wish to thank the Everest ER volunteers over the years and the Himalayan Rescue Association for the use of their documentation.

\section{References}

1. Gallagher SA, Hackett PH. High-altitude illness. Emerg Med Clin North Am. 2004;22:329-355.

2. Hawley E, Salisbury R. The Himalayan Database; The Expedition Archives of Elizabeth Hawley. Golden, CO: American Alpine Club; 2004-2013.

3. West JB, Lahiri S, Maret KH, Peters RM Jr, Pizzo CJ. Barometric pressures at extreme altitudes on Mt. Everest: physiologic significance. J Appl Physiol Respir Environ Exerc Physiol. 1983;54:1188-1194.

4. Freer L. Descriptive report of experience designing and staffing the first-ever medical clinic at Mt. Everest base camp, 2003. High Alt Med Biol. 2004;5:89-90.

5. Basnyat B, Cumbo TA, Edelman R. Acute medical problems in the Himalayas outside the setting of altitude sickness. High Alt Med Biol. 2000;1:167-174.

6. Basnyat B, Savard GK, Zafren K. Trends in the workload of the two high altitude aid posts in the Nepal Himalayas. J Travel Med. 1999;6:217-222.

7. McIntosh SE, Campbell A, Weber D, Dow J, Joy E, Grissom CK. Mountaineering medical events and trauma on Denali, 1992-2011. High Alt Med Biol. 2012;13:275-280.

8. Mason NP, Petersen M, Mélot C, et al. Changes in plasma bradykinin concentration and citric acid cough threshold at high altitude. Wilderness Environ Med. 2009;20:353-358.

9. Mason NP, Barry PW. Altitude-related cough. Pulm Pharmacol Ther. 2007;20:388-395.

10. Shrestha IL, Shrestha SL. Indoor air pollution from biomass fuels and respiratory health of the exposed population in Nepalese households. Int J Occup Environ Health. 2005;11:150-160.

11. Joshi SK, Dudani I. Environmental health effects of brick kilns in Kathmandu valley. Kathmandu Univ Med J (KUMJ). 2008;6:3-11.

12. Kurmi OP, Devereux GS, Smith WC, et al. Reduced lung function due to biomass smoke exposure in young adults in rural Nepal. Eur Respir J. 2013;41:25-30.

13. Fransen M, Pérodin J, Hada J, He X, Sapkota A. Impact of vehicular strike on particulate matter air quality: results from a natural intervention study in Kathmandu valley. Environ Res. 2013;122:52-57.

14. Stephens BD, Diekema DS, Klein EJ. Recreational injuries in Washington state national parks. Wilderness Environ Med. 2005;16:192-197.

15. Forrester JD, Holstege CP. Injury and illness encountered in Shenandoah National Park. Wilderness Environ Med. 2009;20:318-326.

16. Schindera ST, Triller J, Steinbach LS, Zimmermann H, Takala J, Anderson SE. Spectrum of injuries from glacial sports. Wilderness Environ Med. 2005;16:33-37.

17. Schöffl V, Morrison A, Schöffl I, Küpper T. The epidemiology of injury in mountaineering, rock and ice climbing. Med Sport Sci. 2012;58:17-43.

18. Dean AG, Yip R, Hoffmann RE. High incidence of mild acute mountain sickness in conference attendees at 10,000 foot altitude. J Wilderness Med. 1990;1:86-92. 
19. Basnyat B, Lemaster J, Litch JA. Everest or bust: a cross sectional, epidemiological study of acute mountain sickness at 4243 meters in the Himalayas. Aviat Space Environ Med. 1999;70:867-873.

20. Jones BE, Stokes S, McKenzie S, Nilles E, Stoddard GJ. Management of high altitude pulmonary edema in the Himalaya: a review of 56 cases presenting at Pheriche medical aid post $(4240 \mathrm{~m})$. Wilderness Environ Med. 2013;24:32-36.
21. Harirchi I, Arvin A, Vash JH, Zafarmand V. Frostbite: incidence and predisposing factors in mountaineers. $\mathrm{Br} \mathrm{J}$ Sports Med. 2005;39:898-901.

22. Hashmi MA, Rashid M, Haleem A, Bokhari SA, Hussain T. Frostbite: epidemiology at high altitude in the Karakoram mountains. Ann R Coll Surg Engl. 1998;80:91-95.

23. Moore GW, Semple JL. Freezing and frostbite on Mount Everest: new insights into wind chill and freezing times at extreme altitude. High Alt Med Biol. 2011;12:271-275. 